\title{
sciendo
}

\author{
BULGARIAN ACADEMY OF SCIENCES
}

CYBERNETICS AND INFORMATION TECHNOLOGIES • Volume 19, No 1

Sofia $2019 \quad$ Print ISSN: 1311-9702; Online ISSN: 1314-4081

DOI: $10.2478 /$ cait-2019-0006

\section{Hybrid Recommender System via Personalized Users' Context}

\author{
Anthony Nosshi ${ }^{1}$, Aziza Asem ${ }^{1}$, Mohamed Badr Senousy ${ }^{2}$ \\ ${ }^{1}$ Information System Dept., Computers and Information Faculty, Mansoura University, Mansoura, \\ Egypt \\ ${ }^{2}$ Computer and Information System Department, Sadat Academy for Management Sciences, Cairo, \\ Egypt \\ E-mails: Anthony.nosshi@gmail.com_AsemAziza@gmail.com Badr_Senousey_arcoit@yahoo.com
}

Abstract: In movie domain, finding the appropriate movie to watch is a challenging task. This paper proposes a recommender system that suggests movies in cinema that fit the user's available time, location, mood and emotions. Conducted experiments for evaluation showed that the proposed method outperforms the other baselines.

Keywords: Movie recommender, Emotion recommendation, Hybrid recommender system, sentiment analysis, spatio-temporal recommendation.

\section{Introduction}

Recommender systems help users overcome the information overload problem. They can be classified into three main categories: Content-Based (CB), Collaborative Filtering (CF) and Hybrid technique. In CB technique, the system builds a user profile based on his/her preferences, then it finds the best fits to this profile [1]. It is helpful in discovering different users' interests [2]. In CF, the system uses other users' ratings to items to understand what the user would like or dislike [3]. The CF can give recommendations to the user that is different from what the user had seen before [4].

Despite the benefits provided by these methods, they still suffer from many issues. For example, CF suffers from sparsity problem, which causes the recommender system to give poor recommendations [5]. Moreover, the CF also suffers from the cold start problem [6]. CB methods also suffer from problems such as the "over-specialization" problem. This problem means the system recommends similar items to what the user has seen [7].

Today's websites and social media enable users to leave their feedback and opinions to be read publicly [8]. Consequently, this enables researchers to extract the users' interests, mood or contextual information from the social media posts they share publicly. Therefore, this information can be incorporated into a hybrid recommender system to produce a better recommendation [9]. Using a hybrid recommender system which applies both aforementioned recommendation techniques allows incorporating additional sources of information and content to produce better recommendations. Therefore, studying additional information about 
the user helps in customizing the recommendation to every user according to his/her needs [10]. Thus, information such as the user's mood during the day, the time of recommendation, and the location of the user can be very helpful in enhancing the recommendation process [11].

This paper proposes a hybrid recommender system that filters the recommendation list using three phases filtering approach. The recommender is applied to the movies in the cinema's domain. In the first phase, the system determines the current user mood to find the most suitable recommendation. Then, the system in the second phase filters the produced list according to the time and location of the user to recommend him/her the nearest cinema that has the preferred movie (Spatio-Temporal Factors). Finally, the system filters the produced list by determining the emotional factors that match with the user's emotions which he/she got from watching another movie previously, regardless of its genre. The remainder of this paper is organized as follows: Section 2 discusses the state of the art, Section 3 discusses the proposed recommender, Section 4 discusses the results and evaluation and finally, Section 5 discusses the conclusion and future work.

\section{State of the art}

Many studies have been conducted by researchers to enhance the recommendation results by incorporating the user's produced contents, sentiments, locations, and other features as follows:

Q. Y a n g [12] proposed a recommender system that integrated both the domain semantics along with the context information. For that purpose, the author developed an improved content-based model to incorporate them. Though this work took into account only four types of emotions. Additionally, it did not take care of the emotional impression that a user may be willing to have as a result of watching a movie. In addition to ignoring the current user's mood, user's location, and user's time availability. Dix it and $\mathrm{J}$ a in [13] proposed a recommender system that took into account the contextual information of three different categories in order to prepare their data selection and construction. However, this work depended only on three main categories: demographic, semantic and social context and ignored the emotional effect of watching movies. In addition, it missed the spatio-temporal factors and the current user mood. Lu is et al. [14] proposed a system based on semantic web technology. The system considered the location, time and the crowd of people at the place of interest. Though, this work missed taking into consideration the user's mood. They depend on their work on location and time and the place crowd only. A b b a s et al. [15] proposed a hybrid movie recommender system that took into consideration the different users' interests and then provided a recommendation based on the context the users were in. Additionally, they applied their approach to four different contexts and then compared how the system performed with these different four contexts. $\mathrm{Ca}$ i and $\mathrm{Gu}$ [16] proposed a recommender system using a tensor factorization method. Though, their work missed the current user mood, the spatio-temporal factors, and the emotional effect of the movies. Kim et al. [17] proposed a recommender system that integrated convolutional neural network into 
probabilistic matrix factorization. Their approach acquired the contextual information from the document by applying the convolutional neural network to enhance the accuracy of rating prediction. Though, they ignored the spatio-temporal effects on the recommendation, the user mood, and the emotional factors as well. $\mathrm{Y} \mathrm{u}$, Lin and Wang [18] proposed a recommendation framework to alleviate the sparsity problem. To this end, they built a contextual profile for each contextual condition using a co-clustering algorithm. Additionally, they used the expanded preferences in their recommendation system. Though, they ignored the emotional effects on movies and matching the personal user's emotions with the movie emotions. Additionally, they missed the user mood. De ng et al. [19] proposed a recommender system that took into account the user's emotions in different granularity levels and different time windows. In comparison to the proposed work, their work took into consideration only the emotional factors and time. They did not take in consideration the spatial factors. $\mathrm{Z} \mathrm{hou}$ et al. [20] proposed a recommender system that analyzed the movie-poster image and the text description for movies, user ratings, and social relationship. Then, they utilized a random-walk methodology for presenting the recommendation. Though, they ignored the spatio-temporal factors.

In summary, the aforementioned studies missed considering in their recommendation process some or all of the following features: the current user mood, the location and time of the recommendation, and the emotional factors in the time domain.

\section{The proposed recommender}

The proposed algorithm consists of the three main phases, the first phase is responsible for determining the user's mood, the second is responsible for determining the spatio-temporal factors and the final phase is responsible for the emotional constraints as illustrated in Fig. 1.

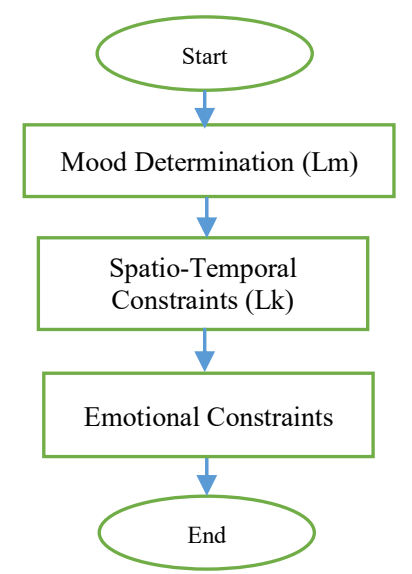

Fig. 1. The proposed recommender overview 


\subsection{The first phase: Mood determination}

The first step in the proposed recommender is to extract the user's mode. In a nutshell, this step consists of two subsystems as illustrated in Fig. 2. The first is (Data Preparation) subsystem, the second is the (Mood Fitting) subsystem.

\subsubsection{The first subsystem: Data preparation}

The goal of this step is to extract the users' emotions from both: The social media posts and the shared movie posts that the user shared in a microblog publicly (Fig. 2). Twitter was used for that purpose to extract users' tweets. Then, some associations between the users, emotions, and movies were constructed and represented in a tuple of three elements (User, Emotion, Movie) as will come in more details in the following paragraphs.

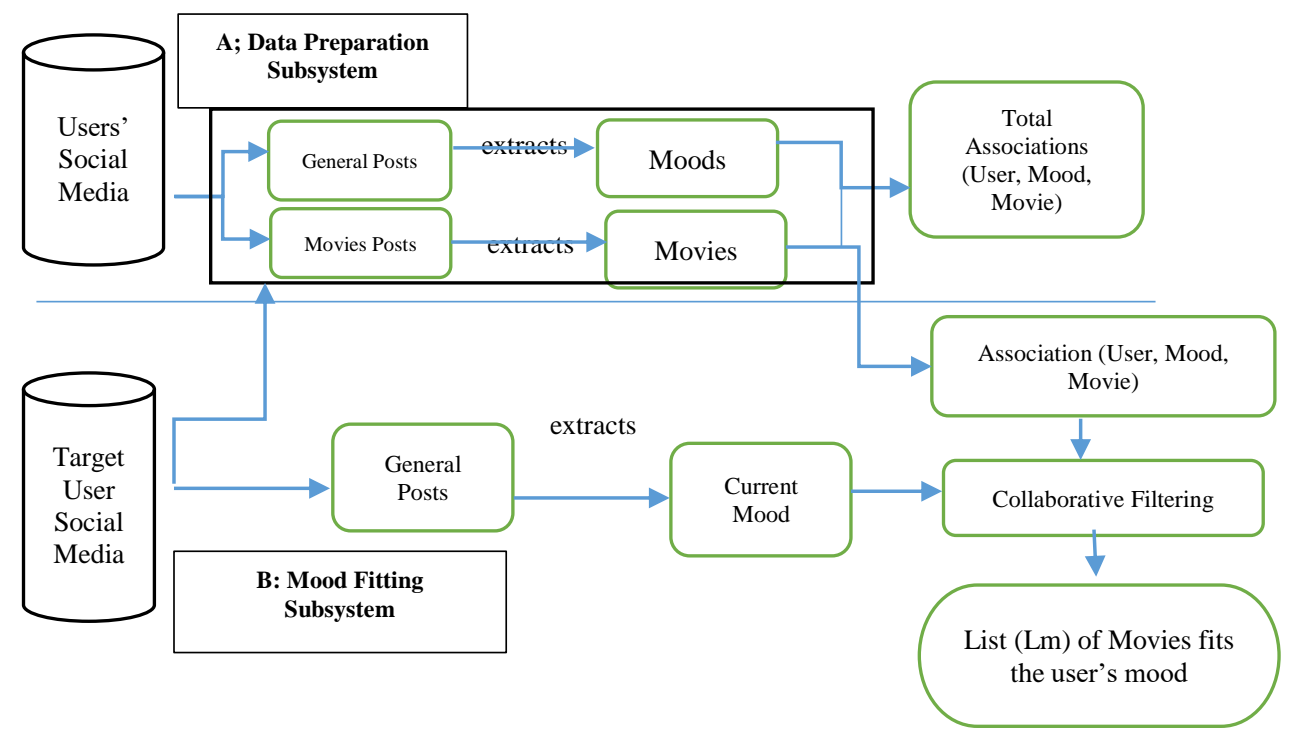

Fig. 2. Mood determinations subsystems

The idea behind the approach applied in this step is that there are associations between the user posts on the social media and the movies they are watching [19]. The emotions expressed in the social media, just before the time that the user expressed him/herself as watching a movie, can reflect to a great extent the mood the user was in to take a decision of watching a specific movie. Applying the same concept represented in [19], the social media posts can be classified into two types: movie related posts, and general posts. The general posts are those, which users usually express everything they want to, such as news, information, personal opinions, etc. While movie posts are those, which the users share to show that, they are watching a movie in their favorite cinema. Table 1 shows the difference between the two types of social media posts. Both the first and the second posts belong to the general post type, while the third belongs to the movie related post. 
Table 1. Microblog example

\begin{tabular}{|c|l|c|c|}
\hline USER ID & Post content & Time & Movie \\
\hline \multirow{2}{*}{876543467} & Would like to thank all my friends for the birthday & $\begin{array}{c}2016.06 .15 \\
13: 25: 39\end{array}$ & \\
& wishes \&... & $\begin{array}{c}2016.06 .15 \\
13: 29: 12\end{array}$ & \\
\hline \multirow{2}{*}{876543467} & Feeling Excited with my friends & $\begin{array}{c}2016.06 .15 \\
13: 55: 02\end{array}$ & Victoria \\
\hline \multirow{2}{*}{876543467} & Watching "Victoria". Best birthday ever with my & lovely friends @ Village East Cinema, 181-189 2nd & \\
& Ave, New York, NY 10003 & & \\
\hline
\end{tabular}

The analysis of the above tweets can infer that the user before he/she has been to the cinema was in a happy mood. He/she was celebrating with their friends by watching a movie called "Victoria". Then, by applying the aforementioned hypothesis, it can be inferred that her decision to watch the movie with his/her friends was according to the correlation between his/her happy mood and the movie they watched. Thus, it was an emotional dependent decision. As illustrated in (Fig. 2), on the first step in data preparation, Twitter posts (tweets) were extracted for people located in the United States using Twitter Enterprise Data (https://developer.twitter.com/en/enterprise). This extraction retrieved $60,345,567$ users' data with an average of 720 tweets per user. Then, users who tweeted less than five movies or cinemas' tweets were filtered out, also movies or cinemas which were tweeted less than five times were removed. The dataset now consists of a total of 12,927,849 tweets. Then we used SAS Viya (https://www.sas.com/en_us/software/viya.html) to extract the sentiments contained in those tweets as it has its own dictionary. Afterwards, all tweet posts were segmented into words that can be used to construct the Bag Of Words model (BOW) [21]. Then, based on that model, the number of emotional words were counted for each emotional class [22] (Granularities of 2d, 7d, and 21d). According to the number of emotional words, the emotional vector of the text was determined. Therefore, each microblog post can be represented into emotional vectors. For example, the first microblog in Table 1 has three emotion vectors: $2 \mathrm{D}$-emotion vector, 7D-emotion vector, and 21D-emotion vector, the values of which are $(3,0),(3,0,0,0,0,0,0)$, and $(3,0,0,0,0,0,0,0,0,0,0,0,0,0,0,0,0,0,0,0,0)$. Then, to discover the emotions and moods related to the movies' posts, only the general social media posts before posting about movies were taken into consideration. For that purpose, the users' latest social media posts were retrieved in the specified time window (last hour in this case). Then, the sum of all qualified social media posts' emotional vectors was calculated. After that, associations were formed between the emotions, movies, and all users. Then, the associations were represented by a three-element tuple (User, Mood, Movie). Example $\quad(876,543,467, \quad((4,0), \quad(3,1,0,0,0,0,0)$, $(3,0,0,1,0,0,0,0,0,0,0,0,0,0,0,0,0,0,0,0,0))$, Victoria)

\subsubsection{The second subsystem: Mood Fitting}

This subsystem (Fig. 2, Mood Fitting) consists of three steps, the first step is for extracting the current user's associations, the second is for calculating the similarity, and the third - for finding out the current user's interest. 
- Step 1. The current user's associations: All the target user's posts (both general and movie related posts) were extracted. Then, the user's current mood and the historical associations were also calculated by the same method discussed in the previous subsystem.

- Step 2. Calculating the similarity: After that, the similarity between the current user and the other users were computed depending on the associations they have by incorporating it into collaborative filtering as presented in [19]. Any two users were considered similar if they watched the same movie before. The similarity degree increased with the more movies being common between the two users. Additionally, if two users watched the same movie and they also shared the same mood, they have more similarity than any other two users who watched the same movie, but under different moods. For example, if three users $\mathrm{A}, \mathrm{B}$ and $\mathrm{C}$ watched the same movie and assuming that $\mathrm{A}$ and $\mathrm{C}$ watched it in a happy mood, and $\mathrm{B}$ watched the same movie in an angry mood, then $\mathrm{A}$ and $\mathrm{C}$ have higher similarity together than B. Then, the users were sorted according to their similarity to the target user and we got the top-k user list.

- Step 3. The current user's interest: Afterwards, the interest of the current user to all movies of the top-k users was calculated as presented in [19] as follows: When the top-k similar users watch a movie in the same current mood of the target user, it is more likely that the user will have a higher interest in that movie. Then, all movies were sorted according to the target user's interest to them and added to a list (denoted here as Lm) to be the input of the next step for spatio-temporal constraints phase.

\subsection{The second phase: Spatio-temporal constraints}

This phase receives the $(\mathrm{Lm})$ list produced from the first phase (Mood Determination) and then applies some processes to produce a filtered movie list that fits with spatiotemporal constraints of the target user. It consists of three main components: User Interface, User Profiler Engine, and Spatio-Temporal engine. It is responsible for determining the spatio-temporal factors. They work as follows.

- User Interface. It is responsible for interacting with both the user and the recommendation engine as well. It is built with Telegram Messenger (Telegram Bot) (https://core.telegram.org/bots). It is also responsible for determining the time and the location of the user using the Google Location API (https://developers.google.com/location-context/fused-location-provider/) (to retrieve the geographical coordinates), and Geocoding API (https://developers.google.com/maps/documentation/geocoding/start) (for reversing geocoding to get a human-readable address). Then, it sends them to the User Profiler Engine to be used in the recommendation process. The time can be either the current time that the user requested the recommendation at, or a time that the user specified according to his/her wish.

- The User Profile Engine. Its job is to build a personal profile for the target user in the system. The profile contains the target user's mood extracted from the first phase, and the retrieved movie list (Lm list), in addition to the time and location retrieved from the user interface. 
- Time Engine. This engine is responsible for filtering out the movie list (Lm) to get the movies' names that are running in cinemas in the user's preferred time. For that purpose, it first retrieves the user profile, which contains the user's preferred time. Then, it applies a programming interface that sends HTTP requests to a movie's API called the International Showtime API (https://api.internationalshowtimes.com) to retrieve a list of movies that are in cinemas at the user's specified time range (date and hour). It also retrieves a list of the cinemas displaying those movies with their details like the address, street, and city. Afterwards, it filters the (Lm) list to pick up the movies and the cinemas in the specified time range, i.e., it discards all the movies that are not in cinemas at the time specified by the user. After filtering the (Lm) list, it creates a new filtered list for movies in a suitable time (denoted here as $\mathrm{Lt}$ ).

- Location Engine. It retrieves the lastly created list (Lt) that contains the movies within the user's preferred time. Then, it uses Google Distance Matrix API (https://developers.google.com/maps/documentation/distance-matrix/start) to retrieve the distance and time needed to reach to the cinemas that display those movies. Therefore, it calculates the distance and the required time for the user to move from the current location (the origin) and the destination cinemas, taking into account the user's mobility method (example: driving, walking, cycling, etc.). Finally, it creates a new filtered list denoted here as (Lk). This list now contains the movies that match the user mood, within her preferable time and in near cinemas to his location. This list of movies (Lk) will be the input of the next third and final step in the recommendation process, the EFBM step.

\subsection{Exploring the emotional factors with emotional fingerprint model}

Now, the prepared movies' list (Lk) is passed to the third phase of the proposed recommender in order to filter the movies for finding which movies can give the user the desired emotions. For that purpose, the Emotional Fingerprints Based Model (EFBM) proposed in [23] was applied. The EFBM model groups movies by emotional patterns of some key factors that change across time, which were extracted from the movies' reviews. Those factors are what people discussed in reviews, showing people's most popular everyday problems, ideas, objects, and philosophical concepts. These patterns in time form a kind of fingerprints that enable the user to choose a movie that can give him/her the same emotional experience he/she got before from watching and liking another movie in the past, regardless of its genre. To apply the EFBM model, the IMDB (https://www.imdb.com/) users' reviews were first extracted for all movies which were included inside the (Lk) list. Then, these customer reviews were analyzed to discover the movies' features and make groups for those movies depending on the features. Afterwards, the algorithm was applied in Fig. 3. 

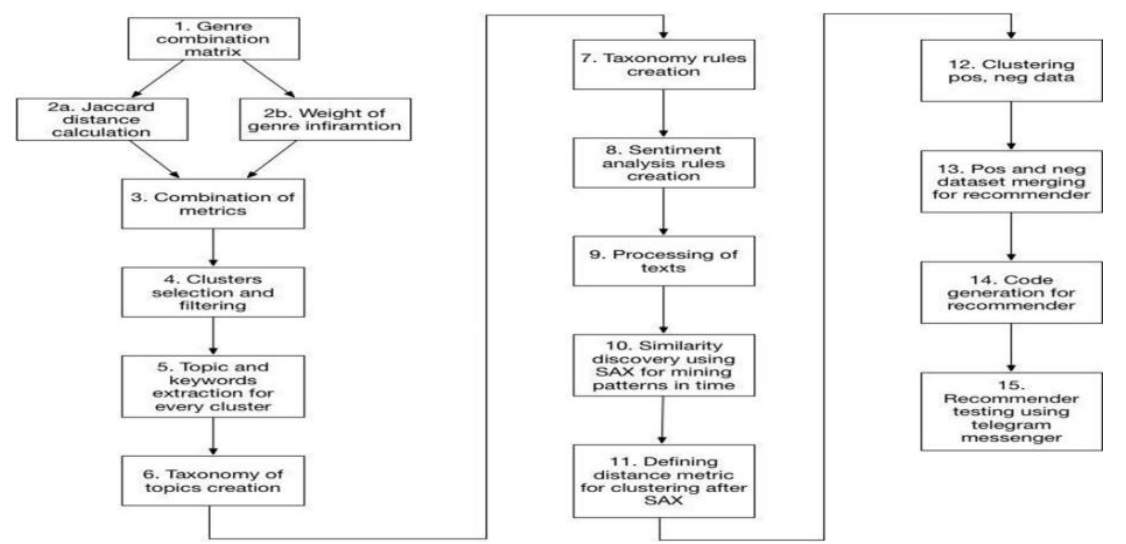

Fig. 3. Algorithm used in the EFBM model [23]

In a nutshell, in the first step of the algorithm, two of small models were created for the data dimension reduction and then aggregated later. After that, the genres were combined with a technique of dimension expanding. The complementary weight was used to modify the Jaccard distance, in order to calculate the value of the information in a genres field. It was calculated as follows [23]:

$$
\mathbf{w}^{\mathbf{w g h}} i j=-\log \left(w_{i}\right) *-\log \left(w_{j}\right),
$$

where $\mathbf{w}^{\mathbf{w g h}}{ }_{i j}$ is the complementary weight; $\boldsymbol{w}_{j}$ is the complementary weight of movie by genre [23], and

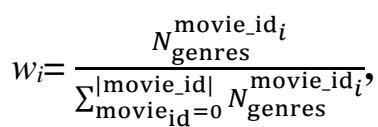

where $N$ is the movie count by all genres. Then, topics were extracted using SAS Enterprise Miner (https://www.sas.com/en_us/home.html) to discover what every cluster is about. After that, the features taxonomy was created manually. Then, the sentiment analysis was applied to each cluster for visualization purposes.
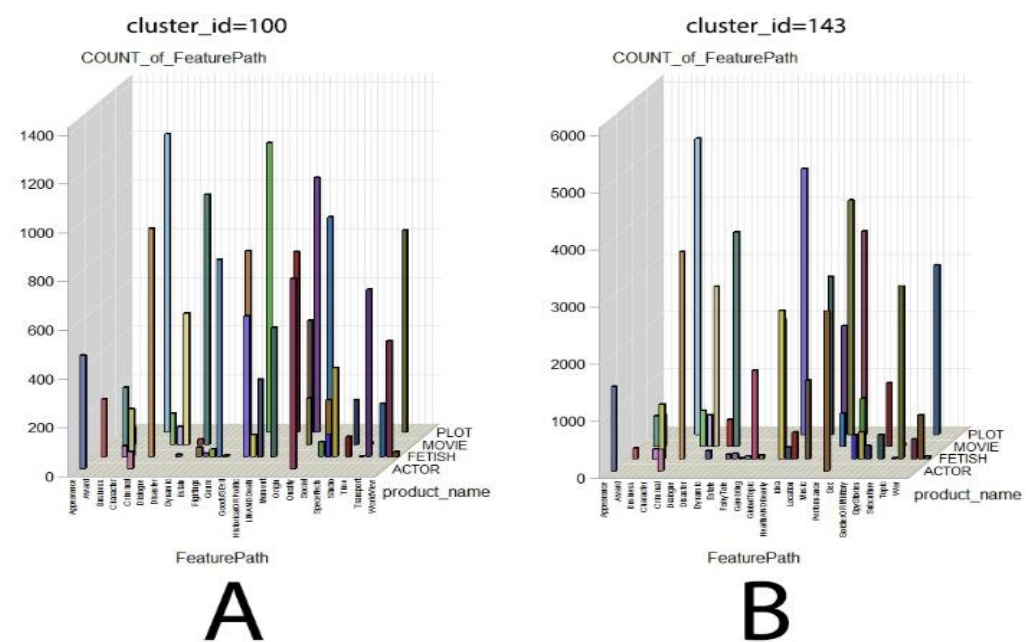

Fig. 4. Statistics of sample clusters after applying sentiment analysis 
Fig. 4 shows the statistics of sample clusters after sentiment analysis was applied (A for cluster_id=100, B for cluster_id=143).

Then, SAX (http://www.cs.ucr.edu/ eamonn/SAX.htm) approach was applied to discover the pattern similarity. SAS was customized with a window of 25 days, intersection of $20 \%$ and size of five alphabets. Then, by applying the distance [23]

$$
\min \left(\left[\left(\operatorname{vec} \_\cos (i, j)\right) \text { for } i, j \text { in } b\right]\right)
$$

a distance matrix was applied for all features to all polarities. Finally, the neutrals were dropped and formed pair by pair by getting the maximum $(\max (a, b))$ to every cell within the matrix. Example of the resulted grouping is given in Fig. 5A, for spy stories - Fig. 5B for War, and Fig. 5C - for Criminal.

A

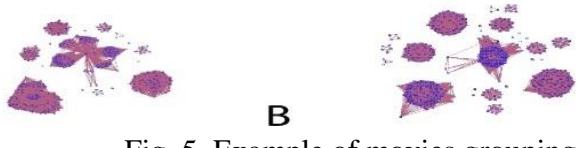

Fig. 5. Example of movies grouping based on the emotional fingerprints

\section{Results and discussion}

To evaluate the proposed approach, the IMDB dataset, which was used in phase three, was split into a training set and a test set using the 10-fold cross validation[24]. Then, the following standard metrics were applied: Precision, Recall, and F-Measure.

In more details, according to [25] Precision measures the ability of the recommender system to retrieve as many relevant items as possible per request. It can be calculated as follows:

$$
\text { Precision }=\frac{\text { Correctly Recommended Items }}{\text { Total Recommended Items }} .
$$

According to [26], a recall measures the system ability to retrieve fewer nonrelevant items as possible. It can be calculated as follows:

$$
\text { Recall }=\frac{\text { Correctly Recommended Items }}{\text { Relevant Items }} .
$$

According to [27], the F-Measures is a harmonic mean of both the precision and recall. It can be calculated as follows:

$$
F \text {-Measure }=\frac{2 \times \text { Precision } \times \text { Recall }}{\text { Precision }+ \text { Recall }} .
$$

Additionally, the experiment was executed on four different user modes, extracted from the social media at the first phase to understand how the recommender is performing with different users' moods. When applying the formulas (4), (5), and (6) over the four different moods, the results were in Table 2.

Table 2. Evaluation metrics for the four moods

\begin{tabular}{|l|c|c|c|c|}
\hline Metric/Mood & Anger & Joy & Sadness & Surprise \\
\hline Precision & 0.89 & 0.99 & 0.84 & 0.97 \\
\hline Recall & 0.85 & 0.96 & 0.77 & 0.91 \\
\hline F-Measure (F1) & 0.87 & 0.97 & 0.80 & 0.94 \\
\hline
\end{tabular}


Table 2 shows the three metrics results with the different four moods. The results show that the proposed model results varied according to the mood the user had. The model achieved a higher precision with the following emotions in the following sequence (Joy, Surprise, Anger, Sadness). Fig. 6 also depicts the different metrics values for the different four moods as well.

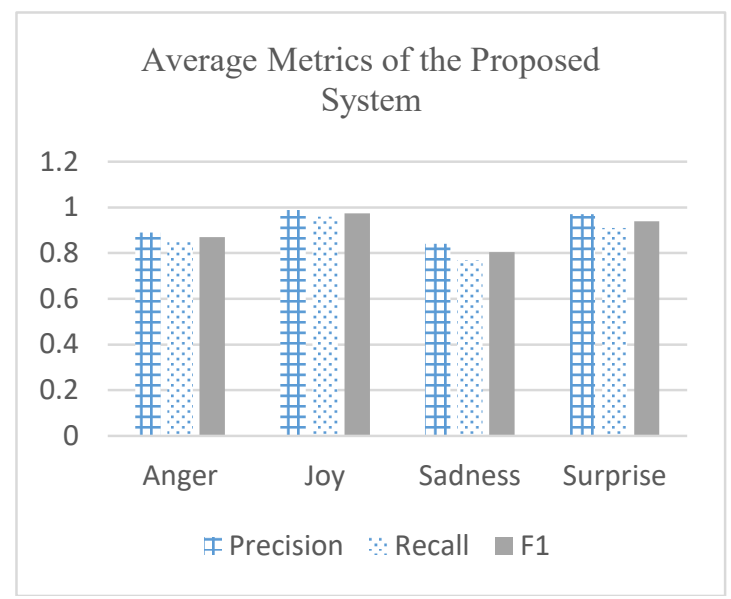

Fig. 6. Average proposed system metrics

As it can be inferred from the figure above, the model achieved different results according to the users' moods. The proposed model achieved better precision results with the Joy mood, then Surprise mood, Anger mood, and lastly Sadness mood.

To evaluate the performance of the proposed recommender, three baselines were used to compare the proposed work with them. The first baseline is by comparing the results of the proposed work with the results of the collaborative filtering technique only. The second baseline is by comparing the proposed work results with the work in [23] which depends on the EFBM only and ignores the mood and location constraints. Finally, the third baseline is by comparing the proposed work results with the work of [15]. In the last work, they applied the experiment where $N=20$. Therefore, in order to be able to compare the proposed work with them, we applied all the metrics where $N=20$ for all the baselines as well. After calculating the aforementioned metrics on the proposed work at $N=20$, we have calculated the average result for those metrics to be able to compare with the other baseline. Table 3 shows the results of the $N=20$ of the four different moods before calculating the average.

Table 3. Evaluation metrics for the four moods at $N=20$

\begin{tabular}{|l|c|c|c|c|}
\hline Metric/Mood & Anger & Joy & Sadness & Surprise \\
\hline Precision & 0.91 & 0.97 & 0.8 & 0.94 \\
\hline Recall & 0.86 & 0.91 & 0.78 & 0.89 \\
\hline F-Measure (F1) & 0.88 & 0.94 & 0.79 & 0.91 \\
\hline
\end{tabular}

Table 3 shows how the results of the three metrics applied at $N=20$ vary according to the user's mood as well. The model had a higher precision result with 
the Joy mood, then Surprise mood, Anger mood and finally the Sadness mood. Fig. 7 also depicts the different metrics values for the different four moods as well.

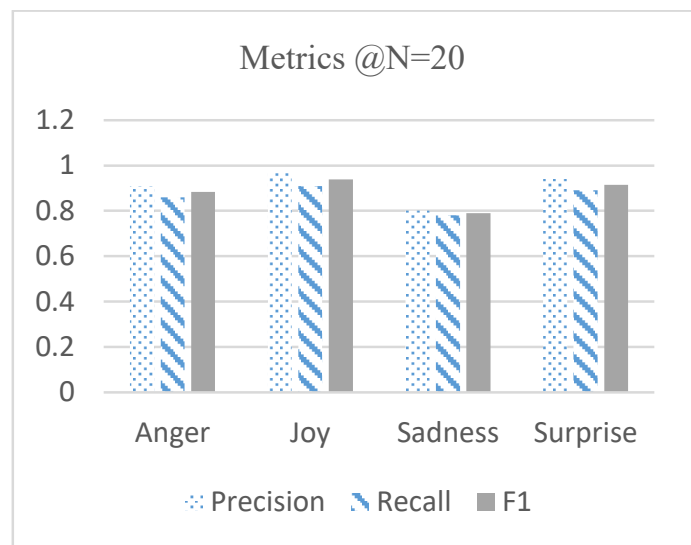

Fig. 7. Evaluation metrics for the four moods at $N=20$

In addition, in work [15] they used the precision metric for evaluation only, therefore we used this metric only in our comparison with the other baselines. Also, they applied the precision metric in four different contexts. Therefore, we calculated their precision average and we also calculated the precision average for our different four moods where $N=20$. Table 4 shows the results of all the baselines where $N=20$.

Table 4. Comparison with Baselines at $N=20$

\begin{tabular}{|c|c|}
\hline Method & Precision \\
\hline Proposed Recommender & 0.92 \\
\hline First Baseline & 0.63 \\
\hline Second Baseline & 0.87 \\
\hline Third Baseline & 0.85 \\
\hline
\end{tabular}

In Table 4, the precision value of the proposed recommender was 0.92 . While it was for the other three baselines as follows: the first baseline $($ Traditional $\mathrm{CF})=63$, the second baseline $(\mathrm{EFBM})=0.87$, while it was for the third baseline (work of $[15])=0.85$. This reflects that the proposed recommender outperformed the other baselines in the precision metric. Fig. 8 also shows a graphical representation of the results.

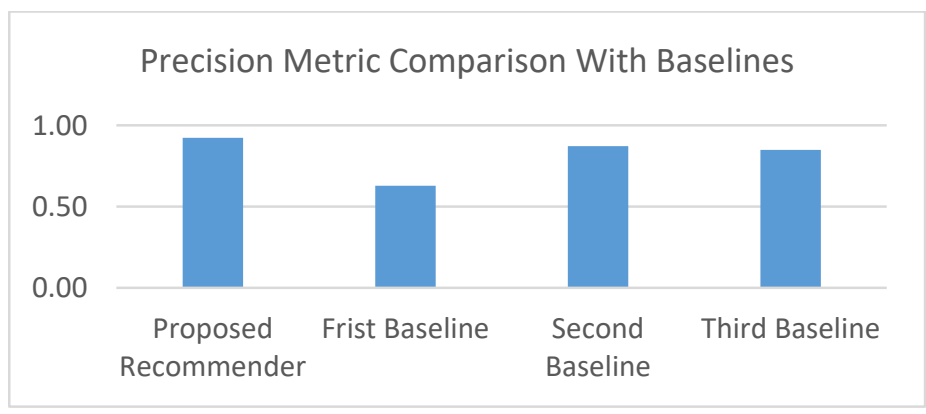

Fig. 8. Precision metric comparison with baselines 
Fig. 8 depicts the results of comparing the results of the proposed recommender with the other baselines. It reflects the superiority of the proposed system against the other baselines.

The experimental results show that using a hybrid recommendation system that takes in consideration the users' moods, location, available time and the emotional features in time, can improve the performance of the recommendation process, which leads to better results and better user satisfaction. The comparison with the results of other baselines shows that the proposed work outperformed the other baselines. This is due to a better understanding of the user's mood, the user's availability, and emotions, which in turn reflects the results of the recommendation, and the user's satisfaction. The average precision for the proposed recommender was 0.92 , while it achieved lower results in other baselines as follows: first baseline $=0.63$, second baseline $=0.87$, and the third baseline $=0.85$. For a deeper understanding of the model, the proposed work was also experimentally tested with four different users' moods, which was (Joy, Sadness, Surprise, and Anger). The experimental results showed that the model best works with the following moods: (Joy, Surprise, Anger, and Sadness) respectively. The results also, to some extent, reflect the nature of human feelings. People can share and post about what they feel in their everyday life especially when they are happy, as it was illustrated on the aforementioned example of Table 1. Whereas when people feel sad, they are usually less expressive about their feelings in social media. This can be due to psychological reasons or simply they are not in a good mood to use the social media that some people still consider it as a means of entertainment.

\section{Conclusion and future work}

The main objective of this paper is creating a hybrid recommender system that takes into consideration many factors such as the user mood, the location, the user's availability, and the user's emotions as well. The proposed recommender consists of three phases. Each phase produces a list of movies that are filtered with other criteria on the next phase. The first phase specifies the user's mood, the next phase specifies the spatio-temporal factors, and on the last phase, the emotional experience is determined by applying the emotional fingerprint-based model.

Experimentations were done to understand the recommendation quality and behavior. The results of the experiment were evaluated using three metrics of evaluation: precision, recall, and F-Measure. In order to understand the quality of the recommendation, the results were compared with three baselines. The first baseline by comparing the results of the proposed model with the results of applying collaborative filtering only. The second baseline is to compare the performance of the upgraded system with the system without upgrade that presented in the previous chapter of this thesis. The third baseline is to compare the experiment with another hybrid recommender system. The comparison showed that the proposed work outperformed all baselines, which reflects the importance of incorporating the user's mood, spatio-temporal aspects, and emotional fingerprint into the recommendation system. 
For future work, more user's context should be taken in consideration such as the weather, seasonality, user's age, gender, job, educational level and more personal details such as marital status, and even her parental status. Additionally, the model should explore what happens if a user was impressed by a specific movie or a feature. In other words, is it possible that the user can dislike a movie while he/she still loves some features, which by the end lead him/her to watch that movie?

Further, adding the emotional factors opens many questions such as: is it still possible that the user changes his/her mind to watch a different movie other than the suggested by the recommender as a result of advertising? Is a feature preferred by a user enough to let him/her watch that movie? Is there any effect for the mass "euphoria" based on a specific topic to lead some people to watch a specific movie or alternate their preferable movie to another one? Example, if some people prefer the movie "Only Lovers Left Alive", which has a strong "life and Death" topic, can people look for some other "life and death" movie such as "Bicentennial Man"? Or just to go by tags "movies by Jim Jarmusch" or "movie with Vampire" as it can be seen on Fig. 9?

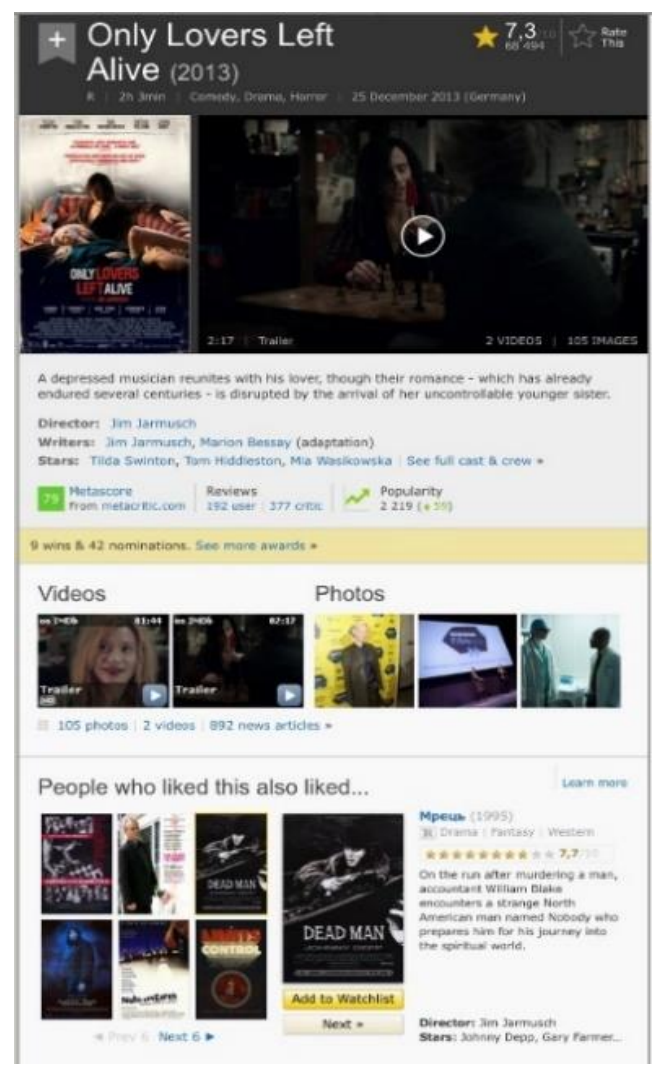

Fig. 9. IMDB proposal for some movies 


\section{References}

1. Cha mpiri, Z. D., S. R. Shah a miri, S. S. B. S alim. A Systematic Review of Scholar Context-Aware Recommender Systems. - Expert Syst. Appl., Vol. 42, 2015, No 3, pp. 1743-1758.

2. S h u, J., X. S h e n, H. Li u, B. Y i, Z. Z h a n g. A Content-Based Recommendation Algorithm for Learning Resources. - Multimed. Syst., Vol. 24, 2018, No 2, pp. 163-173.

3. F u, M., H. Q u, D. M o g e s, L. L u. Attention Based Collaborative Filtering. - Neurocomputing, Vol. 311, 2018, pp. 88-98.

4. W a n g, F., S. Li n, X. L u o, H. W u, R. W a n g, F. Z h o u. A Data-Driven Approach for SketchBased 3D Shape Retrieval via Similar Drawing-Style Recommendation. - Comput. Graph. Forum, Vol. 36, October 2017, No 7, pp. 157-166.

5. Chu, P. M., S. J. Le e. A Novel Recommender System for e-Commerce. - In: Proc. of 10th International Congress on Image and Signal Processing, BioMedical Engineering and Informatics (CISP-BMEI'17), 2017, pp. 1-5.

6. Zh a o, Q., C. W ang, P. W ang, M. Zhou, C. Ji ang. A Novel Method on Information Recommendation via Hybrid Similarity. - IEEE Trans. Syst. Man, Cybern. Syst., Vol. 48, 2018, No 3, pp. 448-459.

7. H a ri a d i, I., D. N u r j a n a h. Hybrid Attribute and Personality Based Recommender System for Book Recommendation. - In: Proc. of International Conference on Data and Software Engineering (ICoDSE'17), 2017, pp. 1-5.

8. Y a n g, S.-B., S.-H. S h i n, Y. J o u n, C. K o o. Exploring the Comparative Importance of Online Hotel Reviews' Heuristic Attributes in Review Helpfulness: A Conjoint Analysis Approach. J. Travel Tour. Mark., Vol. 34, September 2017, No 7, pp. 963-985.

9. W a $\mathrm{n}$ g, H., K. G u o. The Impact of Online Reviews on Exhibitor Behaviour: Evidence from Movie Industry. - Enterp. Inf. Syst., Vol. 11, November 2017, No 10, pp. 1518-1534.

10. Clarizia, F., F. Cola ce, M. Lo mbardi, F. P a s c al e. A Context Aware Recommender System for Digital Storytelling. - In: Proc. of IEEE 32nd International Conference on Advanced Information Networking and Applications (AINA'18), 2018, pp. 542-549.

11. B offa, S., C. D. Maio, B. Gerla, M. Parente. Context-Aware Advertisment Recommendation on Twitter through Rough Sets. - In: Proc. of IEEE International Conference on Fuzzy Systems (FUZZ-IEEE'18), 2018, pp. 1-8.

12. Y a n g, Q. A Novel Recommendation System Based on Semantics and Context Awareness. Computing, Vol. 100, 2018, No 8, pp. 809-823.

13. D i x it, V. S., P. J a in. A Proposed Framework for Recommendations Aggregation in Context Aware Recommender Systems. - In: Proc. of 8th International Conference on Cloud Computing, Data Science \& Engineering (Confluence), 2018, pp. 209-214.

14. Colombo-Mendoza, L. O., R. Valencia-García, A. Rodríguez-González, G. A l or-Hernández, J. J. S a mper-Z a p a ter. RecomMetz: A Context-Aware Knowledge-Based Mobile Recommender System for Movie Showtimes. - Expert Syst. Appl., Vol. 42, 2015, No 3, pp. 1202-1222.

15. A b b a s, M., M. U. Riaz, A. Rauf, M. T. Khan, S. Khalid. Context-Aware Youtube Recommender System. - In: Proc. of International Conference on Information and Communication Technologies (ICICT'17), 2017, pp. 161-164.

16. $\mathrm{Ca}$ i, G., W. Gu. Heterogeneous Context-Aware Recommendation Algorithm with SemiSupervised Tensor Factorization BT. - Intelligent Data Engineering and Automated Learning - IDEAL'17, 2017, pp. 232-241.

17. K i m, D., C. P a r k, J. O h, S. L e e, H. Y u. Convolutional Matrix Factorization for Document Context-Aware Recommendation. - In: Proc. of 10th ACM Conf. Recomm. Syst. (RecSys'16), 2016, pp. 233-240.

18. Y u, P., L. L i n, J. W a n g. A Novel Framework to Alleviate the Sparsity Problem in Context-Aware Recommender Systems. - New Rev. Hypermedia Multimed., Vol. 23, April 2017, No 2, pp. 141-158.

19. Deng, S., D. Wang, X. Li, G. X u. Exploring User Emotion in Microblogs for Music Recommendation. - Expert Syst. Appl., Vol. 42, 2015, No 23, pp. 9284-9293. 
20. Z h a o, Z., et al. Social-Aware Movie Recommendation via Multimodal Network Learning. - IEEE Trans. Multimed., Vol. 20, 2018, No 2, pp. 430-440.

21. Z h a n g, Y., R. J i n, Z.-H. Z h o u. Understanding Bag-of-Words Model: A Statistical Framework. - Int. J. Mach. Learn. Cybern., Vol. 1, 2010, No 1, pp. 43-52.

22. P. Ekman, R. J. Davidson, Eds. The Nature of Emotion: Fundamental Questions. New York, NY, US, Oxford University Press, 1994.

23. N o s s hi, A., A. A s e m, M. B. S e n o u s y. Hybrid Recommender System Using Emotional Fingerprints Model. - Int. J. Inf. Retr. Res., Vol. 9, 2019, No 3, p. Article 4 (in Press).

24. K o h a vi, R. A Study of Cross-Validation and Bootstrap for Accuracy Estimation and Model Selection. - In: Proc. of 14th International Joint Conference on Artificial Intelligence, Vol. 2, 1995, pp. 1137-1143.

25. Y u a n, T., H. W u, J. Z h u, L. S h e n, G. Q i a n. MS-UCF: A Reliable Recommendation Method Based on Mood-Sensitivity Identification and User Credit. - In: Proc. of International Conference on Information Management and Processing (ICIMP'18), 2018, pp. 16-20.

26. Wang, H.-C., H.-T. Jhou, Y.-S. Ts a i. Adapting Topic Map and Social Influence to the Personalized Hybrid Recommender System. - Inf. Sci., NY, 2018.

27. L i m, H., H.-J. K i m. Item Recommendation Using Tag Emotion in Social Cataloging Services. Expert Syst. Appl., Vol. 89, 2017, pp. 179-187.

Received: 29.01.2019; Accepted: 14.02.2019 (fast track) 